individual but also on the family, community and society. Just as the mental health effects on the individual psyche can result in nonpathological distress as well as a variety of psychiatric disorders, massive and widespread trauma and loss can impact on family and social processes as well as the collective unconscious causing changes at the family, community and societal levels.

Methods: This ecological study used qualitative methods of Participatory Action Research in Cambodia and Northern Sri Lanka, while involved in community mental health programmes among the Tamil and Khmer communities. Participant observation, key informant interviews and focus group discussion with community relief and rehabilitation workers and officials were used.

Results: Fundamental changes in the functioning of the family and the community included the dynamics of single-headed families, lack of trust among members, and changes in significant relationships (motherchild), and childrearing practices. Communities were more dependent, passive, silent, without leadership, mistrustful and suspicious. Additional adverse effects included the breakdown in traditional structures, institutions and familiar ways of life, and deterioration in social norms and ethics. On the positive side, the study observed the emergence of community organizations, enhanced female role and leadership, and a decrease in suicide rates.

Conclusions: Exposure to conflict, war and disaster impact on family and community dynamics resulting in changes at a collective level. Relief, rehabilitation and development programmes will need to use integrated multilevel approaches.

\section{Multilevel modeling of rural mental health}

\author{
H Stain', N Higginbotham², T Lewin ${ }^{3}$, B Kelly' ${ }^{1}$ P Lane ${ }^{1}$ \\ 'Centre for Rural and Remote Mental Health, University of Newcastle; \\ ${ }^{2}$ Centre for Clinical Epidemiology and Biostatistics, University of Newcastle; \\ and ${ }^{3}$ Hunter New England Mental Health, Newcastle, Australia
}

Background: The lack of consistent findings regarding comparisons of mental health between rural and urban areas has been attributed in part to methodological shortcomings, including poor conceptualization of rurality. The influence of social environment context (community, family and individual factors) on mental health may be addressed through multilevel modeling. A rural mental health database was developed to address the diversity of rural communities and included data on health, lifestyle, social capital, climate patterns, agricultural activity and primary industry.
Aim: The study sought to investigate the association between mental health, health behaviours and social context in rural communities.

Method: Items from the NSW Health Survey were used, initially across the 37 Divisions of General Practice in New South Wales. The response variable of the percentage of people who had high or very high psychological distress, as measured by the K10, was regressed against social capital items (such as attending community events), health accessibility item (difficulties in accessing health care) and measures of rurality (remoteness, population density and changes in population structure over time).

Results and Conclusions: Associations between psychological distress and measures of health service accessibility, social capital, lifestyle and rural population changes will be reported. Analyses will be extended in a multilevel framework to include important agricultural, meteorological and environmental stress indicators, to assess the effects of drought on psychological distress. This analysis will be conducted using the 176 local government areas in New South Wales and will allow more detailed analysis to examine any heterogeneous effects in rural New South Wales.

\section{Prevalence of mental disorders in the elderly: the Australian National Mental Health and Well-being Survey}

\section{J Trollor, H Brodaty, G Andrews, P Sachdev, T Anderson}

School of Psychiatry, University of New South Wales, Sydney, Australia

Background: We describe 1- and 12 month prevalence of mental disorders, demographic correlates and impact on service utilization and disability in individuals 65 years of age and over in the Australian National Mental Health and Well-being Survey (NMHWS)

Methods: A noninstitutionalized national probability sample of elderly Australian residents was interviewed as part of NMHWS. The prevalence of ICD-10 and DSM-IV mental disorders was estimated from the Composite International Diagnostic Interview and other screening instruments.

Results: Of 1792 elderly NMHWS respondents, 13\% reported symptoms consistent with a mental disorder in the past month, and $16 \%$ reported symptoms consistent with a mental disorder in the past 12 months. Women experienced higher rates of affective disorders and generalized anxiety disorder and had lower rates of substance abuse compared with men. Increasing age was associated with less likelihood of having 